\title{
Sexualidade na terceira idade e terapia comportamental: revisão integrativa
}

\author{
Solemar Lergnani Araújo*, Robson Zazula**
}

\section{Resumo}

A sexualidade durante a terceira idade não é melhor nem pior que a do jovem: simplesmente é diferente. O presente trabalho propõe-se a realizar uma revisão integrativa ressaltando a importância da terapia comportamental na sexualidade dos idosos, principalmente nas disfunções sexuais. $\mathrm{O}$ texto destaca considerações feitas pela pesquisa de campo e também pela revisão de literatura, que utilizou artigos publicados nas bases de dados Lilacs, PubMed e Scielo, nos idiomas português e inglês, publicados no período de 2003 a 2013. Foram utilizados de modo puro ou combinado os seguintes descritores: disfunções sexuais/ terapia comportamental/sexualidade/idoso, e então foram escolhidos treze artigos para serem analisados. Após a análise, concluiu-se que a sexualidade da terceira idade é assunto de grande relevância, que é comum e normal a ocorrência de disfunções sexuais nessa população, e que a terapia comportamental tem se mostrado um eficiente instrumento de tratamento.

Palavras-chave: Idoso. Sexualidade. Disfunção sexual. Terapia comportamental.

\section{Introdução}

A velhice é a última fase da vida, e o envelhecimento está diretamente relacionado a mudanças físicas, psicológicas e sociais (FRAIMAN, 1994). No passado, a sexualidade do idoso foi negada e esquecida; hoje sabemos, por meio de uma infinidade de estudos, todos os benefícios que a sexualidade nessa etapa da vida traz para a saúde, para o bem-estar e para a satisfação geral (GOTT; HINCHLIFF, 2003; PASCUAL, 2002; RISMAN, 1996).

Os idosos estão cada vez mais conscientes de seus direitos e sabem que não podem ser privados do exercício de uma vida saudável e de sua sexualidade em razão da idade. Para qualquer pessoa, a sexualidade torna-se forma de encontro, relação, comunicação ou expressão dos afetos (BUTLER; LEWIS, 1985).

Como qualquer indivíduo, o idoso tem necessidades e desejos sexuais. A sexualidade durante a terceira idade não

* Graduada em Psicologia e especialista em Análise do Comportamento pela Faculdade Guairaca de Guarapuava, PR. Psicóloga no Instituto de Saúde Santa Clara, Candói, PR.

** Graduado em Psicologia. Mestre em Análise do Comportamento pela Universidade Estadual de Londrina. Professor assistente I do curso de Medicina da Universidade Federal da Integração Latino-Americana. Endereço para correspondência: Universidade Federal da Integração Latino-Americana. Instituto Latino-Americano de Ciências da Vida e da Natureza, curso de Medicina. Avenida Tarquino Joslim dos Santos, 1.000, Jardim Universitário - Foz do Iguaçu, PR, CEP 85870-901. E-mail: robson.zazula@unila.edu.br

$\rightarrow$ http://dx.doi.org/10.5335/rbceh.v12i2.5054

Recebido em: 18/05/2015. Aceito em: 05/08/2015 
é melhor nem pior do que a do jovem: simplesmente é diferente. $\mathrm{O}$ grande tumulto emocional que caracteriza a vida sexual da terceira idade decorre principalmente de dois fatores: o primeiro é a falta de conhecimento das modificações fisiológicas que ocorrem na atividade sexual do indivíduo, e o segundo são as pressões culturais (LERNER, 2012; BAGGIO, 1990).

Ser idoso não é sinônimo de doença e enfermidade. A sexualidade para o idoso constitui um fator muito importante para gozar de uma saúde integral. Ela é fonte de satisfação e não há porque deixar de ser nessa fase da vida, pois sua suspensão ou abandono pode acelerar o processo de envelhecimento e repercutir, assim, negativamente na saúde do idoso (PASCUAL, 2002).

O presente trabalho pode ser considerado importante devido à necessidade de se compreender e tratar sobre essa temática. Os indivíduos, ao se depararem com uma idade mais avançada, necessitam de uma vida ativa, não sendo desprezados e inúteis para a família e/ ou para a sociedade. Compartilhando da mesma opinião de Pascual (2002), justifica-se a necessidade de apoiar e incentivar os idosos quanto à prática da sexualidade no decurso da vida, independentemente de limites, disfunções sexuais e dificuldades. É importante priorizar os benefícios de uma qualidade de vida associada à vida sexual ativa por meio da terapia comportamental.

Não há idade para o sexo, uma vez que as barreiras são socioculturais. A ideia de que sexo é privilégio dos jovens e que não pode fazer parte da idade madura é equivocada. A sexualidade é uma forma de expressão que está presente em todo ser humano e não deve ser diferente na velhice, não sendo só o ato sexual, mas sim um momento de intimidade que deve ser vivenciado pelo idoso (PASCUAL, 2002). Dessa forma, objetiva-se, com o presente trabalho, descrever, por meio de uma revisão integrativa, estudos sobre terapia comportamental na sexualidade dos idosos bem como a importância dessa prática, especialmente em relação às disfunções sexuais.

\section{Método}

O presente trabalho caracteriza-se por ser uma revisão integrativa de literatura, baseada em artigos publicados nas bases de dados Lilacs, PubMed e Scielo. O levantamento foi realizado no período de dezembro de 2013 a fevereiro de 2014. Foram pesquisadas publicações do período de 2003 a 2013, utilizando de modo puro ou combinado os seguintes descritores: disfunções sexuais/terapia comportamental/sexualidade/idoso. Após a seleção dos artigos, os critérios de exclusão foram: artigos científicos que não fossem em português ou em inglês, estar fora do período selecionado e não abordar a temática em questão.

Para a realização da pesquisa, optou-se pelo método de revisão integrativa da literatura. A pesquisa integrativa, segundo Souza, Silva e Carvalho (2010), consiste na incorporação das evidências na prática clínica, segundo a qual se escolhe um tema, pesquisa-se diferentes aspectos sobre ele $\mathrm{e}$, de forma sistemática e ordenada, produz-se um conhecimento fortemente embasado. Constitui um 
estudo de diversas publicações sobre o tema proposto em determinado período, incluindo estudos experimentais e não experimentais, com o objetivo de realizar uma síntese com conclusões gerais sobre a área. Além disso, objetiva identificar falhas e limitações dos estudos bem como sugerir novas questões e temas para pesquisa nesta temática.

Foi utilizado um instrumento validado para a coleta das informações, elaborado por Oliveira (2010) e adaptado para este estudo, contemplando as seguintes informações: a) título do artigo; b) autoria do artigo; c) categoria do profissional dos autores; d) periódico de publicação; e) ano de publicação; f) método utilizado para o estudo; g) objetivos do estudo; h) principais resultados encontrados.

\section{Resultados}

Ao longo da pesquisa nas bases de dados, foram encontrados mais de cem artigos abordando a temática proposta para o estudo. Após a aplicação dos critérios de exclusão, foram selecionados treze artigos para análise e discussão. Esses artigos foram analisados de modo quantitativo e qualitativo, por meio da descrição de questões relacionadas à categoria profissional dos responsáveis pelo estudo, período e ano de publicação, principais objetivos delimitados e principais resultados encontrados (Quadro 1).
Quadro 1 - Artigos analisados na revisão

Artigos analisados na revisão

1. ABDOLL, C. H. N.; FLEURY, H. J. Aspectos diagnósticos e terapêuticos das disfunções sexuais femininas. Revista de Psiquiatria Clínica, São Paulo, v. 3, n. 33, p. 162-167, 2006.

2. CALDAS, C. P. et al. Terapia comportamental para incontinência urinária da mulher idosa: uma ação do enfermeiro. Texto \& Contexto - Enfermagem, Florianópolis, v. 19, n. 4, p. 783-788, 2010.

3. SILVA, L. A. et al. Abordagem das disfunções sexuais femininas. Revista Brasileira de Ginecologia e Obstetrícia, Rio de Janeiro, v. 30, n. 3, p. 12-21, jun. 2008.

4. SOUSA, J. L. Sexualidade na terceira idade: uma discussão da Aids envelhecimento e medicamentos para disfunção erétil. DST - Jornal Brasileiro de Doenças Sexualmente Transmissíveis, Niterói, RJ, v. 20, n. 1, p. 59-64, 2008.

5. VACANTI, L. J.; CARAMELLI, B. Idade e distúrbios psicológicos: variáveis associadas à disfunção sexual no período pós-infarto. Arquivos Brasileiros de Cardiologia, São Paulo, v. 85, n. 2, p.110-114, ago. 2005.

6. FLEURY, H. J.; ABDOLL, C. H. N. Envelhecimento, doenças crônicas e função sexual. Diagnóstico e Tratamento, São Paulo v. 17, n. 4, p. 201-205, out.-dez. 2012.

7. VASCONCELOS, D. et al. A sexualidade no processo do envelhecimento: novas perspectivas - comparação transcultural. Estudos de Psicologia (Natal), Natal, RN, v. 9, n. 3, p. 413-419, set.-dez. 2004.

8. LIDÓRIO, A. A.; TATAREN, J. C. Disfunções sexuais masculinas. Psicologia Clínica na Análise do Comportamento, Londrina, v. 18. p. 1-18, 2008.

9. ALMEIDA, T.; LOURENÇO, M. L. O comportamento sexual na terceira idade, saúde sexual para o idoso e a questão da Aids (terceira idade e a Aids). In: PESSÔA, C. V. B. B.; COSTA, C. E.; BENVENUTI, M. F. (Org.). Comportamento em foco. São Paulo: Associação Brasileira de Psicologia e Medicina Comportamental, 2011. p. 19-30.

10. BATISTONI, S. S. T. Contribuições da psicologia do envelhecimento para as práticas clínicas com idosos. Psicologia em Pesquisa - UFJF, Juiz de Fora, MG, v. 3, n. 2, p. 13-22, jul.-dez. 2009.

11. FÁVERO, M. F.; BARBOSA, S. C. S. Sexualidade na velhice: os conhecimentos e as atitudes dos profissionais de enfermagem. Terapia Sexual, São Paulo, v. 14, n. 2, p. 11-40, jul.-dez. 2011.

12. DUTRA, D. V. M.; REIS, M. A disfunção do desejo sexual feminino e a terapia comportamental. Revista de Psicologia, Belo Horizonte, v. 2, n. 2, p. 1-2, jul. 2010.

13. SERAPIÃO, J. J.; RIBEIRO JUNIOR, J. Psicoterapia na Disfunção eretil. Jornal Brasileiro de Medicina, v. 86, p. 42-48, 2004.

Fonte: dos autores. 
Conforme se pode acompanhar, cinco estudos abordam a questão das disfunções sexuais em mulheres, dois estudos abordam as disfunções sexuais entre os homens, dois abordam a sexualidade e sua interface com doenças crônicas, dois abordam questões culturais bem como suas inter-relações com a sexualidade na terceira idade, e um aborda diretamente o papel e o comportamento do profissional de saúde em relação à temática com pacientes idosos.

Uma das características que podem ser observadas por meio dos títulos é a diversidade de áreas de atuação dos autores principais dos trabalhos. Uma das informações que foram levantadas por meio das revisões foi a categoria profissional dos autores do estudo. Dos treze estudos analisados, cinco foram publicados por profissionais de psicologia, cinco por profissionais de medicina, dois por equipes multidisciplinares e apenas um por enfermeiros. Essas informações estão descritas na Tabela 1.

Tabela 1 - Quantidade de artigos publicados segundo a categoria profissional

\begin{tabular}{l|r}
\hline \multicolumn{1}{c|}{ Categoria profissional } & $\begin{array}{c}\text { Quantidade de } \\
\text { artigos }\end{array}$ \\
\hline Médico & 5 \\
Psicologia & 5 \\
Equipe multidisciplinar & 2 \\
Enfermagem & 1 \\
\hline Fonte: dos autores.
\end{tabular}

Em relação ao ano de publicação dos estudos, três artigos foram publicados em 2008, três em 2011 e dois artigos em 2010. Os anos de 2004, 2005, 2006, 2009 e 2012 tiveram apenas uma publicação relacionada ao tema. Em relação ao método, nove artigos foram de revisão de literatura, quatro foram pesquisas de campo, dessas, três foram quantitativas e uma qualitativa. Houve ainda um estudo de revisão narrativa. Dentre os principais objetivos dos artigos de revisão de literatura, quatro objetivaram identificar na literatura questões relacionadas ao tratamento das disfunções sexuais na terceira idade, dois revisavam estudos que abordavam a sexualidade na terceira idade, um artigo buscava identificar na literatura as causas e prevalências das disfunções sexuais na terceira idade e outro abordava a temática do comportamento sexual na terceira idade. Pode-se acompanhar o método bem como o principal objetivo de cada artigo no Quadro 2. 
Quadro 2 - Métodos e objetivos dos artigos analisados

\begin{tabular}{|c|c|c|}
\hline Artigo & Método & Objetivos \\
\hline 1 & Revisão de literatura & Tratar das classificações das disfunções sexuais. \\
\hline 2 & $\begin{array}{l}\text { Pesquisa de campo } \\
\text { - quantitativa }\end{array}$ & $\begin{array}{l}\text { Relatar uma experiência de implementação da terapia comportamental para } \\
\text { incontinência urinária na mulher idosa. }\end{array}$ \\
\hline 3 & Revisão de literatura & $\begin{array}{l}\text { Levantar as causas e a prevalência das disfunções sexuais e oferecer es- } \\
\text { tratégias de abordagem da função sexual feminina. }\end{array}$ \\
\hline 4 & Revisão de literatura & $\begin{array}{l}\text { Apresentar uma revisão da literatura científica atual, abordando os temas } \\
\text { da sexualidade na terceira idade, medicamentos para disfunção erétil e a } \\
\text { tendência crescente de Aids entre idosos. }\end{array}$ \\
\hline 5 & $\begin{array}{l}\text { Pesquisa de campo } \\
\text { - quantitativa }\end{array}$ & $\begin{array}{l}\text { Avaliar pacientes de ambos os sexos, com vida sexual ativa e sem dis- } \\
\text { função sexual prévia ao infarto do miocárdio, para estudar a incidência de } \\
\text { disfunção sexual após o infarto do miocárdio, e identificar as possíveis vari- } \\
\text { áveis associadas a elas. }\end{array}$ \\
\hline 6 & Revisão narrativa & $\begin{array}{l}\text { Apresentar um levantamento das alterações na função sexual próprias do } \\
\text { envelhecimento e de doenças crônicas. }\end{array}$ \\
\hline 7 & $\begin{array}{l}\text { Pesquisa de campo } \\
\text { - quantitativa }\end{array}$ & $\begin{array}{l}\text { Caracterizar conhecimentos, atitudes, crenças e práticas sobre a vivência } \\
\text { do corpo e da sexualidade de } 187 \text { pessoas entre } 52 \text { e } 90 \text { anos, gozando de } \\
\text { boa saúde física e mental. }\end{array}$ \\
\hline 8 & Revisão de literatura & $\begin{array}{l}\text { Elencar os transtornos sexuais masculinos bem como a definição e a pre- } \\
\text { valência de cada um deles. }\end{array}$ \\
\hline 9 & Revisão de literatura & $\begin{array}{l}\text { Discutir sobre o comportamento sexual na terceira idade, a saúde sexual e } \\
\text { a questão da Aids. }\end{array}$ \\
\hline 10 & Revisão de literatura & $\begin{array}{l}\text { Trazer uma revisão de alguns avanços que a temática do envelhecimento } \\
\text { proporcionou ao saber psicológico a partir da última metade do século XX, } \\
\text { principalmente no que se refere à consolidação do campo teórico da psico- } \\
\text { logia do envelhecimento. }\end{array}$ \\
\hline 11 & $\begin{array}{l}\text { Pesquisa de campo } \\
\text { - qualitativa }\end{array}$ & $\begin{array}{l}\text { Identificar os conhecimentos dos profissionais de enfermagem em face de } \\
\text { sexualidade na velhice; verificar se os profissionais de enfermagem abor- } \\
\text { dam esse tema na educação para a saúde ao idoso; avaliar as necessida- } \\
\text { des de formação, no que tange à sexualidade, dos profissionais de enfer- } \\
\text { magem. }\end{array}$ \\
\hline 12 & Revisão de literatura & $\begin{array}{l}\text { Demonstrar a relevância da terapia comportamental no tratamento de uma } \\
\text { das disfunções sexuais mais presentes em consultórios, a disfunção do de- } \\
\text { sejo sexual. }\end{array}$ \\
\hline 13 & Revisão de literatura & $\begin{array}{l}\text { Analisar a atuação do ginecologista diante das queixas femininas, conside- } \\
\text { rando as diversas etapas da vida da mulher sexualmente madura. }\end{array}$ \\
\hline
\end{tabular}

\section{Discussão}

Ao se analisar os estudos de Abdoll e Fleury (2006) e Caldas et al. (2010), que abordam as disfunções sexuais femininas, e no caso do estudo de Caldas et al. a incontinência urinária, observa-se que são casos muito frequentes, mesmo que muitas mulheres não procurem ajuda profissional. São estudos de revisão de literatura e de pesquisa de campo, e os dois confluem em um mesmo pensamento, ou seja, que as disfunções sexuais femininas, em sua maioria, não são de ordem orgânica, mas sim psíquica (i.e., por estado de estresse, depressão, fadiga, ansiedade, vergonha). Pode-se observar que a terapia comportamental é uma 
das estratégias usadas que restabelece o equilíbrio emocional da paciente, favorecendo o tratamento das disfunções.

De certa forma, cada indivíduo constrói sua velhice e, quando ela chega, vive aquilo que construiu. Com isso, pode-se dizer que a sexualidade da pessoa idosa é, em grande parte, um reflexo de sua história de vida em relação à sexualidade. $\mathrm{O}$ desejo e a necessidade sexual continuam preservados ao longo da terceira idade, porque a idade não emudece sexualmente ninguém (BUTLER; LEWIS, 1985). Tais questões são evidenciadas nos estudos de Abdoll e Fleury (2006) e Caldas et al. (2010).

Outro estudo de Fleury e Abdoll (2012), que se constitui em uma revisão narrativa, também retrata o envelhecimento e as disfunções sexuais. Os autores concluíram que há alta prevalência de comorbidades de doenças em homens idosos e que há associação dessa condição com o comprometimento da função sexual. Isso confirma o prejuízo do interesse e da satisfação sexual entre essa população. O mesmo acontece com as mulheres idosas. De acordo com esses autores, elas relatam aumento de dor durante a penetração e a diminuição do desejo sexual. Os autores afirmam que, para o tratamento dessa população específica com disfunção sexual, há necessidade de uma abordagem mais adequada por parte dos profissionais de saúde, avaliando as condições biológicas, psicossociais e afetivas.

De acordo com Liberalesso (2001), a sexualidade envolve questões biológicas, sociais e psicológicas. No aspecto biológico, desde os 40 anos os homens apre- sentam mudanças fisiológicas, pois há redução da produção de testosterona e, em algumas pessoas, são desencadeados sintomas psicológicos como depressão e irritabilidade. A melhor maneira de prevenir o problema é cuidar da saúde mental e física ao longo da vida. O que muda com o passar dos anos é o intervalo entre uma ereção e outra, mas o que se perde em quantidade, pode ser revertido em qualidade. Tal mudança está relacionada à forma como o idoso encara as mudanças decorrentes do envelhecimento, bem como sua história e experiência de vida (RISMAN, 1996). As mulheres, após a menopausa, apresentam lubrificação vaginal menos intensa e de mais demorado aparecimento, evento que pode simplesmente ser corrigido com o uso de lubrificantes locais. Os orgasmos, embora mais curtos, têm a mesma intensidade daqueles experimentados pelas mulheres mais jovens. De qualquer forma, é necessário reconhecer que cada um tem uma maneira própria de expressar sua sexualidade (LIBERALESSO, 2001).

Entretanto, diversas mudanças acontecem em todas as esferas da vida do idoso. O grande tumulto emocional que caracteriza a vida sexual do homem na terceira idade decorre de dois fatores principais: o primeiro é a falta de conhecimento das modificações fisiológicas que ocorrem na atividade sexual do indivíduo, e o segundo são as pressões culturais (LIBERALESSO, 2001; RISMAN, 1996).

Com relação à Aids e à sexualidade na terceira idade, Sousa (2008) e Almeida e Lourenço (2011) destacam, em seus artigos de revisão de literatura, que a vida sexual do indivíduo transforma-se 
constantemente. Isso não ocorre de modo diferente com os idosos. É consenso entre os autores que existem alguns fatores que afetam o comportamento sexual. São eles: saúde física, preconceitos sociais e econômicos, autoestima, conhecimentos sobre a sexualidade e o status conjugal. Atualmente, discute-se muito sobre a Aids em todas as idades. Pesquisas indicam que indivíduos entre 50 e 70 anos de idade tornaram-se mais suscetíveis à Aids nos últimos anos (ALMEIDA; LOURENÇO, 2011; SOUSA, 2008), por falta de esclarecimento, valores culturais, sociais e econômicos e, principalmente, por não utilizarem preservativos.

De acordo com Almeida e Lourenço (2011), no que se refere à sexualidade na terceira idade, no Brasil, é necessário reavaliar as estratégias de cuidados e as políticas públicas sociais de saúde no âmbito da família e da sociedade. É importante estimular uma prática saudável e sem estigmas, e um dos tratamentos que se destaca é na área de psicologia, com as psicoterapias (NERI, 2002). Além disso, é importante intervir adequadamente diante de uma série de problemas e distúrbios, especialmente as disfunções sexuais, independentemente do gênero.

Nesse caso, destacam-se dois estudos da área médica: o de Silva et al. (2008) e o de Serapião e Ribeiro Júnior (2004). Esses autores realizaram revisões de literatura com o objetivo de fazer uma abordagem refinada sobre as disfunções sexuais femininas e seus tratamentos. Destacam que as disfunções são comuns nas diferentes faixas etárias, especialmente após a menopausa, destacando-se o desejo sexual hipoativo (DSH). Dentre os tratamentos destacados por eles está o modelo Pilset, que oferece uma abordagem ampla envolvendo as queixas de origem biológica ou psíquica e aquelas referentes ao desconhecimento da anatomia e da resposta sexual. Além disso, pode-se oferecer como tratamento a farmacoterapia, com diversas restrições, e o uso da psicoterapia intensiva e da terapia de casal com relações conflituosas e/ou agressivas.

Outro estudo que merece destaque é o de Vacanti e Caramelli (2005), que trata sobre os distúrbios psicológicos e a disfunção sexual no período pós-infarto. Tanto na literatura como nesse estudo, percebe-se que a doença coronariana geralmente atinge a mulher em faixa etária maior que o homem, resultando em maior morbimortalidade. Nesse estudo, todos os participantes apresentaram significativa redução da frequência da atividade sexual e elevada incidência de disfunção sexual após o infarto agudo do miocárdio, com destaque para o gênero masculino. Além disso, mais enfaticamente, observou-se que a presença de distúrbios psicológicos e a idade mais elevada estiveram associadas à maior incidência de disfunção sexual após o infarto.

De acordo com Butler e Lewis:

Se o idoso teve que se abster de sexo por razão médica durante certo período de tempo, alguns medicamentos serão necessários quando a atividade sexual for retomada. A estimulação sexual irregular ou pouco frequente pode interferir com o funcionamento sexual saudável, afetando negativamente a potência masculina e a lubrificação, o formato vaginal e a tonicidade muscular na mulher. Estas dificuldades provavelmente irão desaparecer quando a atividade for retomada e não se deve desencorajar diante das dificuldades iniciais. Quando um parcei- 
ro sexual não está presente (como por exemplo, na viuvez) ou quando as circunstâncias não permitem que se mantenha contato com o parceiro, tanto os homens como as mulheres podem proteger grande parte de sua capacidade sexual através da masturbação regular, a menos que pessoalmente isso seja inaceitável (1985, p. 39).

Os estudos de Batistoni (2009) e de Vasconcelos et al. (2004) buscaram investigar outras facetas acerca da temática do envelhecimento e da sexualidade. Batistoni (2009) salienta alguns temas fundamentais para o estudo do envelhecimento: adaptação, autonomia e dependência, regulação emocional e qualidade de vida. Os estudos acerca do envelhecimento têm contribuído de forma contundente às práticas psicológicas. Evidências atuais de pesquisas têm demonstrado que idosos são responsivos às intervenções psicológicas mais diversas.

Atualmente, discute-se sobre a perspectiva Life-Span, que deixa de lado a ideia de que o idoso é um ser passivo e doente, reforçando a importância de atividades para a manutenção do envelhecimento saudável. Considera-se assim o desenvolvimento um processo contínuo. Dessa forma, faz-se necessário que os psicólogos estejam familiarizados com os problemas e a vida diária dos idosos para que possam usar de forma adequada as psicopatologias e metodologias de avaliação válidas e confiáveis para essa população. Com relação à ansiedade, à sexualidade e aos problemas de adicção (i.e., vícios), percebeu-se maior efetividade de abordagens cognitivo-comportamentais (BATISTONI, 2009).

Essa proposta está em sintonia com os pressupostos básicos da Organização das Nações Unidas (ONU, 2003). De acordo com esses pressupostos, é necessário que os cidadãos que chegam à terceira idade tenham disposição emocional para manter uma vida sexual ativa e viver melhor. Tais questões estão intimamente relacionadas à qualidade de vida dos indivíduos.

O estudo realizado por Vasconcelos et al. (2004) buscou comparar brasileiros e portugueses quanto à sexualidade em idosos, com maior participação feminina nessa pesquisa. De modo geral, as mulheres brasileiras declararam estar muito mais interessadas e sexualmente mais ativas que as portuguesas, e também muito mais livres quanto à comunicação verbal com o cônjuge acerca da sexualidade. Além disso, pôde-se perceber que os adultos maduros manifestam interesse pela sexualidade e têm consciência da sua receptividade ao prazer sexual. Entretanto, existe uma elevada frequência de recusa de respostas sobre as práticas sexuais. Para os autores, faz-se necessária uma maior demanda de conhecimentos sobre o funcionamento fisiológico e psicológico dessa faixa etária para sua melhor abordagem.

No que se refere à terapia comportamental e à sua interface com a sexualidade, especialmente durante a terceira idade, o estudo de Lidório e Tataren (2008) caracteriza-se por ser uma revisão bibliográfica em que se discute as disfunções sexuais masculinas. De acordo com os autores, deve-se adotar a etiologia dos transtornos sexuais segundo o modelo biopsicossocial, envolvendo os fatores biológicos, psicológicos e sociais, com destaque para os fatores psicológicos, isto é, 
comportamentos modelados e mantidos ao longo da história de vida do indivíduo. Quando pensamos em problemas de cunho sexual, é essencial considerar tais questões. Dentre os transtornos mais comuns que ocorrem com os homens, segundo esses autores, estão o desejo sexual hipoativo, o transtorno erétil e a ejaculação precoce.

$\mathrm{O}$ tratamento indicado, de acordo com Lidório e Tataren (2008), é a psicoterapia, por meio de uma estratégia multimodal, que envolve a entrevista clínica, os questionários de autorrelato bem como os procedimentos que envolvem o comportamento respondente. Os autores ressaltam a importância de uma avaliação psicológica cuidadosa e recomendam que o(a) parceiro(a) participe da avaliação.

Para a análise do comportamento, o repertório sexual e os operantes são estabelecidos por influências genéticas, ambientais e culturais (LIDÓRIO; TATAREN, 2008). O operante é o comportamento mais provável na presença de um estímulo discriminativo devido a uma história de reforço na sua presença. A maneira como as primeiras relações sexuais ocorreram, o reforçamento (e.g., orgasmo e ejaculação), dentre outros, definirá classes de respostas operantes, que, dependendo de suas consequências, poderão ou não ser mantidas no repertório do indivíduo. Por isso a adoção da terapia comportamental é importante para o tratamento de disfunções sexuais tanto masculinas quanto femininas.

Por fim, é importante destacar o papel do profissional da saúde e como ele pode lidar com questões que envolvam a sexualidade na terceira idade.
Fávero e Barbosa (2011) abordaram em seu estudo a sexualidade na velhice e os conhecimentos e as atitudes dos profissionais de enfermagem. Esse artigo foi escolhido, embora não trate especificamente de psicologia, porque sua importância se dá em explicar de forma mais clínica como realmente é a sexualidade entre os idosos, quais os inconvenientes e a real importância de se manter sexualmente ativo no decorrer do seu desenvolvimento.

Em relação aos conhecimentos dos enfermeiros sobre o tema, percebeu-se que apresentam conhecimentos médios sobre a sexualidade na velhice. Além disso, alguns apresentam mitos e ideias erradas sobre o tema. Os profissionais reconhecem a sua falta de conhecimento e que essa seria a razão pela qual não abordam temas relacionados à sexualidade com idosos. Embora a terapia comportamental seja uma importante estratégia para lidar com problemas e dificuldades ligados à atividade sexual e à sexualidade, observam-se poucos estudos que adotam tal estratégia durante seu atendimento. No que concerne à formação profissional, conclui-se que ela não lhes proporciona preparação adequada para lidar com a sexualidade ao longo do ciclo vital, especialmente durante a terceira idade.

\section{Considerações finais}

O tema sexualidade e envelhecimento merece atenção especial, pois o envelhecimento traz consigo inúmeras mudanças orgânicas, físicas e psicológicas, que estão intimamente interligadas com a vida sexual do indivíduo, e o idoso, assim como 
qualquer outro indivíduo, merece ter uma vida sexual que o satisfaça.

Com a realização deste estudo, percebe-se que o tema sexualidade e envelhecimento deve ser mais estudado pelos diversos profissionais da área da saúde. Tais estudos devem visar conhecimentos adequados para atender de forma satisfatória seu público-alvo, especialmente na área de psicologia.

Embora a terapia comportamental seja uma importante estratégia para lidar com problemas e dificuldades decorrentes ligadas à atividade sexual e à sexualidade, observam-se poucos estudos que adotam tal estratégia. $\mathrm{O}$ acervo acerca do assunto em questão ainda é limitado, especialmente em nosso país, quando se leva em consideração a atenção que ele merece.

Além disso, sabe-se do inevitável aumento da população idosa brasileira para os próximos anos, uma vez que há baixos níveis de natalidade e elevada expectativa de vida. Por essa razão, sugere-se a realização de novos estudos empíricos bem como revisões de literatura sobre a temática. Tais estudos poderão contribuir para a melhor compreensão do tema e proporcionar melhor qualidade de vida à população idosa.

\section{Sexuality in the elderly and behavioral therapy: an integrative review}

\section{Abstract}

Sexuality in the elderly is not better or worse than in the younger one: it is simply different. This study aims to conduct an integrative review, highlighting the importance of behavioral therapy on sexuality of the el- derly, especially in sexual dysfunctions. The author points out considerations set out for field survey as well as literature review. This study used articles published in Lilacs, PubMed and Scielo database, with Portuguese and English language, published in the period 2003-2013. The following descriptors were used on pure or combined mode: Sexual Dysfunctions/Behavioral Therapy/ Sexuality/Elderly and then 13 articles were selected for analysis. After analysis, it was concluded that the sexuality of the elderly is a matter of great importance that is common and normal to experience sexual dysfunctions and that the behavioral therapy has proved to be an efficient tool for treatment.

Keywords: Elderly. Sexuality. Sexual dysfunction. Behavioral therapy.

\section{Referências}

ABDOLL, C. H. N.; FLEURY, H. J. Aspectos diagnósticos e terapêuticos das disfunções sexuais femininas. Revista de Psiquiatria Clínica, São Paulo, v. 3, n. 33, p. 162-167, 2006.

ALMEIDA, T.; LOURENÇO, M. L. Amor e sexualidade na velhice: direito nem sempre respeitado. Revista Brasileira de Ciências do Envelhecimento Humano (RBCEH), Passo Fundo, v. 5, n. 1, p. 130-140, jan./jun. 2008

O comportamento sexual na terceira idade, saúde sexual para o idoso e a questão da Aids (terceira idade e a Aids). In: PESSÔA, C. V. B. B.; COSTA, C. E.; BENVENUTI, M. F. (Org.). Comportamento em foco. São Paulo: Associação Brasileira de Psicologia e Medicina Comportamental, 2011. p. 19-30.

BAGGIO, M. A. Aspectos psicológicos da sexualidade do idoso. Revista Intercâmbio, São Paulo, v. 3, n. 79, p. 27-34, jan.-dez. 1990.

BATISTONI, S. S. T. Contribuições da psicologia do envelhecimento para as práticas clínicas com idosos. Psicologia em Pesquisa - UFJF, Juiz de Fora, MG, v. 3, n. 2, p. 13-22, jul.-dez. 2009. 
BUTLER, R. N.; LEWIS, M. I. Sexo e amor na terceira idade. São Paulo: Summus, 1985.

CALDAS, C. P. et al. Terapia comportamental para incontinência urinária da mulher idosa: uma ação do enfermeiro. Texto \& Contexto - Enfermagem, Florianópolis, v. 19, n. 4, p. 783-788, 2010.

DUTRA, D. V. M; REIS, M. A disfunção do desejo sexual feminino e a terapia comportamental. Revista de Psicologia, Belo Horizonte, v. 2, n. 2, p. 1-2, jul. 2010.

FÁVERO, M. F.; BARBOSA, S. C. S. Sexualidade na velhice: os conhecimentos e as atitudes dos profissionais de enfermagem. Terapia Sexual, São Paulo, v. 14, n. 2, p. 1140, jul.dez. 2011.

FLEURY, H. J.; ABDOLL, C. H. N. Envelhecimento, doenças crônicas e função sexual. Diagnóstico e Tratamento, São Paulo, v. 17, n. 4, p. 201-205, out.dez. 2012.

FRAIMAN, A. P. Sexo e afeto na terceira idade. São Paulo: Gente, 1994.

GOTT, M.; HINCHLIFF, S. How important is sex in later life? The views of older people. Social Science and Medicine, v. 56, p. 617628, 2003.

LERNER, T. Terapia cognitivo-comportamental em grupo de disfunções seuxuais femininas. 2012. Dissertação (Mestrado em Obstetrícia e Ginecologia) - Faculdade de Medicina, Universidade de São Paulo, São Paulo, 2012.

LIBERALESSO, A. (Org.). Desenvolvimento e envelhecimento: perspectivas biológicas, psicológicas e sociológicas. Campinas: $\mathrm{Pa}-$ pirus, 2001.

LIDÓRIO, A. A.; TATAREN, J. C. Disfunções sexuais masculinas. Psicologia Clínica na Análise do Comportamento, Londrina, v. 18, p. 1-18, 2008.

NERI, A. L. (Org.). Cuidar de idosos no contexto da família: questões psicológicas e sociais. Campinas: Alínea, 2002.
OLIVEIRA, A. Revisão integrativa sobre a consulta de enfermagem: enfoque das abordagens e modelagens de educação em saúde evidenciadas. 2010. Trabalho de conclusão de curso (Graduação em Enfermagem) - Escola de Enfermagem, Universidade Federal do Rio Grande do Sul, Porto Alegre, 2010.

ONU. ORGANIZAÇÃO DAS NAÇÕES UNIDAS. Plano de ação internacional contra o envelhecimento, 2002. Brasília: Secretaria Especial dos Direitos Humanos, 2003.

PASCUAL, C. P. A sexualidade do idoso vista com novo olhar. São Paulo: Loyola, 2002.

RISMAN, A. Sexualidade e o homem idoso. Arquivos de Geriatria e Gerontologia, Rio de Janeiro, v. 10, n. 1, p. 123-124, 1996.

SERAPIÃO, J. J.; RIBEIRO JUNIOR, J. Psicoterapia na Disfunção eretil. Jornal Brasileiro de Medicina, v. 86, p. 42-48, 2004.

SILVA, L. A. et al. Abordagem das disfunções sexuais femininas. Revista Brasileira de Ginecologia e Obstetrícia, Rio de Janeiro, v. 30, n. 3, p. 12-21, jun. 2008.

SOUSA, J. L. Sexualidade na terceira idade: uma discussão da Aids envelhecimento e medicamentos para disfunção erétil. $D S T$ - Jornal Brasileiro de Doenças Sexualmente Transmissíveis, Niterói, RJ, v. 20, n. 1, p. 59-64, 2008.

SOUZA, M. T.; SILVA, M. D.; CARVALHO, R. Revisão integrativa: o que é e como fazer. Einstein, São Paulo, v. 8, n. 1, p. 102-106, 2010.

VACANTI, L. J.; CARAMELLI, B. Idade e distúrbios psicológicos: variáveis associadas à disfunção sexual no período pós-infarto. Arquivos Brasileiros de Cardiologia, São Paulo, v. 85, n. 2, p. 110-114, ago. 2005.

VASCONCELOS, D. et al. A sexualidade no processo do envelhecimento: novas perspectivas - comparação transcultural. Estudos de Psicologia, Natal, RN, v. 9, n. 3, p. 413-419, set.dez. 2004.

RBCEH, Passo Fundo, v. 12, n. 2, p. I72-182, maio/ago. 2015 